\title{
The Effect of Die Bearing Geometry on Surface Recrystallization During Extrusion of an Al-Mg-Si-Mn Alloy
}

\author{
Y. MAHMOODKHANI, J. CHEN, M.A. WELLS, W.J. POOLE, and N.C. PARSON
}

The formation of large surface grains known as peripheral coarse grains (PCG) is an undesirable feature commonly observed in extruded medium-strength Al-Mg-Si alloys produced for many applications including automotive. The objective of this study was to evaluate factors contributing to the formation of PCG layers, particularly the die bearing geometry, with the goal of developing strategies to eliminate or reduce this phenomenon. This was accomplished using a combination of extrusion trials and finite element method simulations to characterize the role of die bearing geometry on the formation of surface microstructure during the extrusion of an Al-Mg-Si-Mn alloy. The extrusion trials were conducted using two die bearing geometries, (i) a zero bearing die and (ii) a choke die using an extrusion temperature of $480{ }^{\circ} \mathrm{C}$ and ram velocities of 20 to $30 \mathrm{~mm} / \mathrm{s}$. Axisymmetric extrusion was conducted with an extrusion ratio of 16.5. During the extrusion trials, partially extruded billets were extracted from the extrusion press and water quenched in order to follow the evolution of the surface microstructure for the different bearing geometries. In addition, ram motion was arrested in the middle of the extruded length, held for 5 seconds and then resumed to investigate the role of changing the deformation conditions of the surface on the extruded microstructure. Optical microscopy and electron backscattered scanning diffraction (EBSD) were used to quantify the microstructure and crystallographic texture of the extrudates and partially extruded billets at different spatial locations. A finite element (FE) mathematical model using the commercial software package DEFORM 2D was also developed to simulate the extrusion process so that loads, temperatures, and material flow patterns could be predicted. The FE model was used to track material flow streamlines close to the surface. Specific locations along these streamlines were then selected for EBSD analysis on the partially extruded billets. The results indicate that the major factor affecting the formation of the PCG layer is the local stored energy of the near surface material which in turn is a function of the details of the die bearing geometry.

https://doi.org/10.1007/s11661-019-05437-0

(C) The Author(s) 2019

\section{INTRODUCTION}

LOCALIZED recrystallization on the surface of an extrudate, known as a Peripheral Coarse Grain (PCG) layer, is a common occurrence during extrusion of medium- to high-strength extruded Al-Mg-Si-Mn alloys such as AA6082. The PCG layer is known to degrade

Y. MAHMOODKHANI and M.A. WELLS are with the Mechanical and Mechatronics Engineering Department, University of Waterloo, 200 University Avenue West, Waterloo, ON N2L3G1, Canada. Contact e-mail: y3mahmoo@uwaterloo.ca J. CHEN and W.J. POOLE are with the Department of Materials Engineering, The University of British Columbia, 309-6350 Stores Road, Vancouver, BC V6T1Z4, Canada. N.C. PARSON is with Rio Tinto Aluminum, Arvida Research and Development Center, Saguenay, QC G7S4L2, Canada.

Manuscript submitted February 6, 2019.

Article published online August 30, 2019 properties such as surface quality, strength, fracture toughness, stress corrosion susceptibility, fatigue resistance, and machinability. ${ }^{[1-3]}$ It has been shown that the formation and severity of the PCG layer depend on alloy composition, extrusion temperature, extrusion ratio, and the homogenization practice. ${ }^{[1,4,5]}$ Typically, the formation and thickness of the PCG layer are exacerbated by higher extrusion temperatures, faster velocities, higher extrusion ratios, slower cooling rates after extrusion, and the use of alloys which do not form dispersoids (i.e., $\mathrm{Mn} / \mathrm{Cr}$ bearing precipitates 20 to 100 $\mathrm{nm}$ in size formed during homogenization). ${ }^{[6]}$

The mechanism responsible for the formation of the PCG layer is still not fully developed but theories point to the characteristics of the surface layer where a combination of very large shear deformation (equivalent strains $>10$ ) and high deformation temperatures (near the melting point of the alloy) develop due to the friction between the extrudate and the die. For example, van 
Geertruyden et al. ${ }^{[4]}$ de Peri and Misiolek ${ }^{[2]}$ proposed that a very fine equiaxed grain structure is produced at the surface by Continuous Dynamic Recrystallization (CDRX) or Geometric Dynamic Recrystallization (GDRX). It was hypothesized that this microstructure was highly unstable, with a high local stored energy and a high degree of heterogeneity which under the right conditions could undergo abnormal grain growth to produce large surface grains.

On the other hand, Eivani ${ }^{[3]}$ proposed that the key processing parameter responsible for the formation of the PCG layer in Al-Mg-Zn alloys (AA7020) is temperature. For example, in this work, no evidence of a PCG layer was found with an extrusion temperature of $460{ }^{\circ} \mathrm{C}$ and a ram velocity of $5 \mathrm{~mm} / \mathrm{s}$, but PCG was observed for extrusion temperatures above $500{ }^{\circ} \mathrm{C}$ using the same ram velocity. Further, Charit and Mishra ${ }^{[7]}$ studied the formation of abnormal grains in friction stir welding of $\mathrm{Al}-\mathrm{Mg}-\mathrm{Zn}$ (7xxx) alloys and suggested that it was the dissolution of $\mathrm{Mg}-\mathrm{Zn}$ containing precipitates which would allow recrystallization/grain growth to occur by reducing the local Smith-Zener drag from the particles. In summary, it is clear that the formation of a PCG layer on the surface of aluminum extrusion alloys is complex as it depends on (i) the driving pressure for recrystallization from the local stored energy, (ii) the thermal conditions (temperature and time) during and after deformation, and (iii) the retarding pressure from second phase particles.

One aspect of the extrusion process which has not received much attention in relation to PCG formation is the die bearing geometry. Jarrett and Parson ${ }^{[8]}$ registered a patent in 1995 covering the use of a choked extrusion die, where there was a small reduction in the billet diameter over a length of several millimeters at the die exit, to suppress surface recrystallization. Based on this finding, Duan and Sheppard ${ }^{[9]}$ studied the effect of the length and degree of taper of the choke using a combination of finite element calculations and a recrystallization model to predict the occurrence of recrystallization near the surface of the extrudate with some success. It was shown that the addition of choke to the die geometry affected the details of the local strain history/microstructure development and increased the temperature of the extrudate surface suggesting that temperature on its own was not the only factor affecting the formation of the PCG layer. Parson et al..$^{[10]}$ examined 5 different die bearing geometries including zero bearing, $12 \mathrm{~mm}$ choked bearing, $25 \mathrm{~mm}$ flat bearing, and $35 \mathrm{~mm}$ choked bearing and showed that the use of a choked die exit geometry could suppress the formation of the PCG layer compared to a conventional flat-faced die. The zero bearing exhibited the most recrystallization and the $35 \mathrm{~mm}$ choked bearing showed the least recrystallization after extrusion.

The current research combines novel experimental extrusion trials and use of a detailed finite element model to gain insight into the generation of the surface microstructures as a function of die bearing geometry during extrusion of an Al-Mg-Si-Mn alloy (AA6082). In this research, axisymmetric extrusion was performed using two limiting die bearing geometries; a so-called zero bearing die which has a minimal contact length with the exiting extrudate and choke die where the extrudate undergoes a small reduction in an area near the exit after the majority of the shape change has occurred. A particular novelty of the extrusion trials was the combination of using an alloy with a high density of dispersoids to suppress recrystallization as much as possible and either (i) quenching the billet and extrudate part way through the extrusion or (ii) stopping the extrusion press, holding and then restarting the extrusion process to capture the evolution of microstructure . The resulting microstructures were characterized using Electron Back-scattered Diffraction (EBSD) and optical microscopy. The effect of die bearing geometry on the thermomechanical history experienced by the material was examined using a previously developed and validated finite element method model ${ }^{[11]}$ and the results were compared to the EBSD observations to gain insight into the near surface deformation conditions and PCG layer formation.

\section{EXPERIMENTAL METHODOLOGY}

\section{A. Material}

Table I shows the chemical composition of the Al$\mathrm{Mg}-\mathrm{Si}-\mathrm{Mn}$ alloy studied. Billets of $400 \mathrm{~mm}$ length and $101.6 \mathrm{~mm}$ diameter were Direct Chill (DC) cast, homogenized for 2 hours at $550{ }^{\circ} \mathrm{C}$ and then extruded into rods of $25 \mathrm{~mm}$ diameter. The microstructure after homogenization for this same alloy has recently been characterized by Liu. ${ }^{[12]}$ It consists of $\alpha$-phase constituent particles characterized by an average radius of $400 \mathrm{~nm}$ with a volume fraction of $1.05 \mathrm{pct}$ and $\alpha$-phase dispersoids which have an average radius of $\approx 40 \mathrm{~nm}$ and a volume fraction of 0.7 pct.

\section{B. Extrusion Trials}

Figure 1 shows the geometries for the zero bearing and choke dies which were selected based on the extremes of PCG tendency observed in the previous work by Parson et al..$^{[10]}$

A 780-tonne extrusion press located at the RioTinto Arvida Research and Development Center in Saguenay, Quebec with a container of $106 \mathrm{~mm}$ diameter was used to extrude the homogenized billets to $25 \mathrm{~mm}$ round bars. The initial billet temperature was $480{ }^{\circ} \mathrm{C}$ and the die and container were pre-heated to $480{ }^{\circ} \mathrm{C}$. An extrusion ram velocity of $20 \mathrm{~mm} / \mathrm{s}$ was initially used. For each trial, three billets were extruded to warm up the press and to reach a steady-state temperature distribution, following which the trial billets were extruded. The extrudates

Table I. Al-Mg-Si-Mn Alloy Composition in Weight Percent (Balance is Aluminum)

\begin{tabular}{ccccc}
\hline $\mathrm{Mg}$ & $\mathrm{Si}$ & $\mathrm{Mn}$ & $\mathrm{Cr}$ & $\mathrm{Fe}$ \\
\hline 0.71 & 1.03 & 0.50 & $<0.01$ & 0.21 \\
\hline
\end{tabular}


were quenched in a standing wave water tank which was located approximately $2 \mathrm{~m}$ from the extrusion press (see Figure 2). During the extrusion of the last billet in each trial, the ram was stopped after about 75 pct of the billet had been extruded and the partially extruded billet was removed and quenched in water to preserve the extruded microstructure. This operation took approximately 2 to 3 minutes to complete. Finally, specific extrusion trials were conducted involving a "dead" stop where the ram motion was arrested for 5 seconds and then was rapidly accelerated back to the original velocity. Note, for these trials a steady-state extrusion ram speed of $30 \mathrm{~mm} / \mathrm{s}$ was used in order to examine a wider range of ram speed during the transient of slowing down, stopping, and resuming. The process conditions and bearing geometry used for the extrusion trial are shown in Table II. All extrusion trials were performed using 60-mm diameter feeder plate. This is a hollow plate located between the container and the die which has an inner diameter smaller than the container and larger than the extrudate

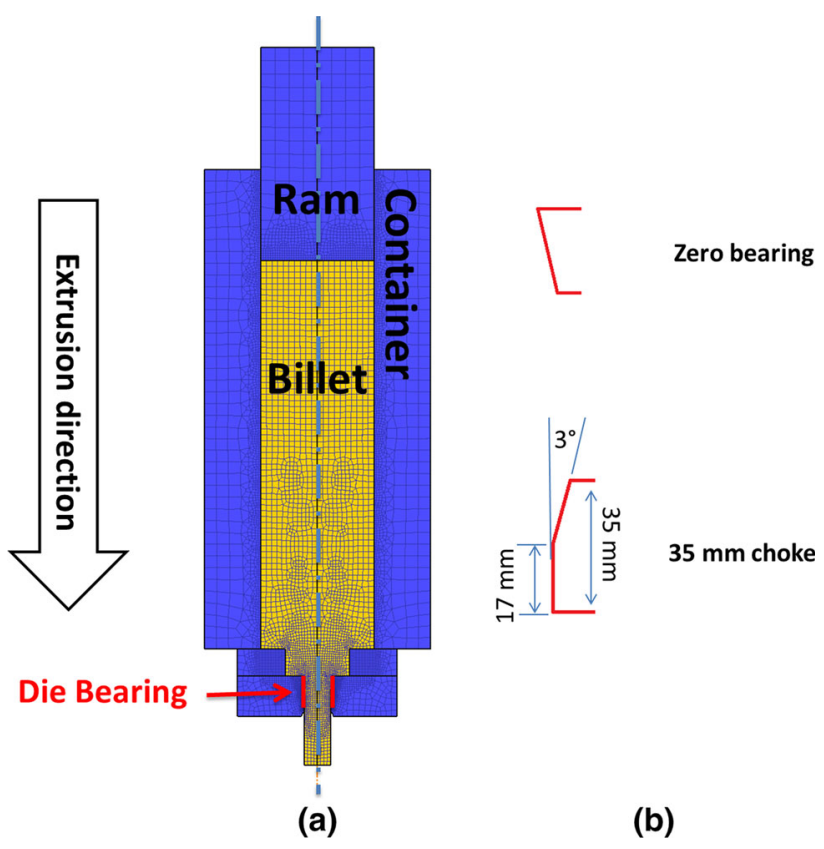

Fig. 1-Schematic of $(a)$ the extrusion press including finite element mesh and $(b)$ die bearing geometries used in this study. and provides a welding chamber to join successive extrusions to permit continuous extrusion.

\section{Metallographic Sample Preparation}

Metallographic samples, cut from the front and back locations as indicated in Figure 2 along with specific locations of interest from the partially extruded billets, were ground and polished before EBSD analysis. Sample preparation included grinding on standard silicon carbide $(\mathrm{SiC})$ paper from grade \#200 to \#1200, polishing using $3 \mu \mathrm{m}$ diamond cloth and final polishing using OPS colloidal silica suspension. Polarized light microscopy was used to observe the grain structure after anodizing in Barker's reagent. Full sections of the partially extruded billets were also cut from the billet centerline and macro-etched after grinding with Poulton's reagent $(2 \mathrm{~mL} \mathrm{HF}, 3 \mathrm{~mL} \mathrm{HCl}, 20 \mathrm{~mL}$ HNO3, 175 $\mathrm{mL}$ water) to reveal the bulk macrostructure.

\section{EBSD Analysis}

EBSD measurements were conducted and analyzed using a Zeiss Sigma scanning field emission electron microscope-EBSD system with EDAX/TSL OIM software. A step size of $1 \mu \mathrm{m}$ was used to reveal the general grain structure and texture, and a step size of $0.3 \mu \mathrm{m}$ was used to reveal details such as subgrain structures. To clean the EBSD maps and remove points with low confidence, all maps were first processed by the Confidence Index (CI) Standardization, which is a function in OIM Analysis. Subsequently, pixels with a CI less than 0.1 were removed.

\section{E. Mathematical Model}

The commercial finite element (FE) package, DEFORM-2D was used to numerically model the effect of die bearing geometry. The FE method used in DEFORM 2D is based on the flow formulation approach using an Updated Lagrangian procedure. The FE model and its validation were described in detail in a previous paper by Mahmoodkhani et al. ${ }^{[13]}$ The first step in the post-processing analysis of the FE results was to track different points across the bar diameter through the velocity field from the billet into the final extruded bar. The spatial locations of the tracked points were selected so that the entire cross-

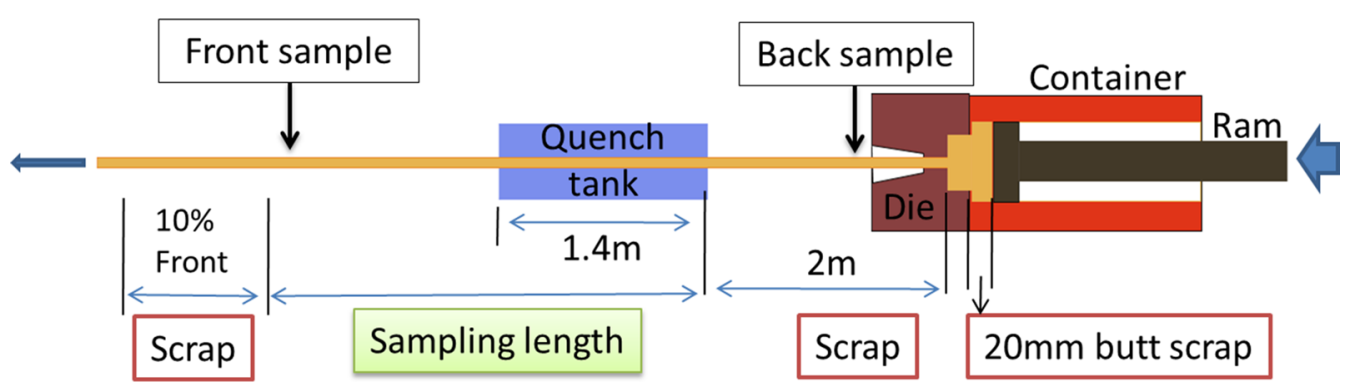

Fig. 2-Schematic view of extrusion set-up showing extrusion press/tooling, quench tank, and location of front and back samples. 
Table II. Process Conditions for Extrusion Trials

\begin{tabular}{|c|c|c|c|c|}
\hline Trial \# & $\begin{array}{c}\text { Billet, Container, } \\
\text { and Die Temperature } \\
\left({ }^{\circ} \mathrm{C}\right)\end{array}$ & $\begin{array}{l}\text { Steady-State Ram } \\
\text { Velocity }(\mathrm{mm} / \mathrm{s})\end{array}$ & Die Type (Bearing) & Description \\
\hline 1 & 480 & 20 & zero bearing & $\begin{array}{l}\text { normal and partial extrusions ( } 100 \mathrm{~mm} \text { butt } \\
\text { end removed and quenched) }\end{array}$ \\
\hline 2 & 480 & 20 & $35 \mathrm{~mm}$ choke & $\begin{array}{l}\text { normal and partial extrusions (100 } \mathrm{mm} \text { butt } \\
\text { end removed and quenched) }\end{array}$ \\
\hline 3 & 480 & 30 & zero bearing & $\begin{array}{l}\text { "dead Stop" extrusion (ram stopped and } \\
\text { accelerated back to steady-state velocity) }\end{array}$ \\
\hline 4 & 480 & 30 & $35 \mathrm{~mm}$ choke & $\begin{array}{l}\text { "dead Stop" extrusion (ram stopped and } \\
\text { accelerated back to steady-state velocity) }\end{array}$ \\
\hline
\end{tabular}

section of the extruded bar was included and these were then back-tracked into the original billet. Back-tracking was performed using the DEFORM-2D post-processor point tracking tool. ${ }^{[13]}$ The thermomechanical history of the tracked points was then extracted in terms of strain, strain rate, and temperature history from which other parameters such as the shear strain and stored energy could be calculated.

Figure 3 shows the geometry and the mesh used in the FE models for each of the die bearing geometries studied.

Based on the previous work performed by $\mathrm{Liu}^{[12]}$ the Al-Mg-Si-Mn alloy was considered as a visco-plastic material and the flow stress of the material as a function of temperature and strain rate was described using the following equation ${ }^{[14]}$ :

$$
A[\sin h(\alpha \sigma)]^{n}=\dot{\varepsilon} \exp \left(\frac{Q_{\mathrm{d}}}{R T}\right)
$$

where $\sigma$ is the flow stress (MPa), $\dot{\varepsilon}$ is the strain rate $\left(\mathrm{s}^{-1}\right)$, and $\mathrm{T}$ is the temperature $(\mathrm{K}) . A, \alpha, n$, and $Q_{\mathrm{d}}$ are material constants with values of $9.04 \times 10^{8} \mathrm{~s}^{-1}, 0.03$ $\mathrm{MPa}^{-1}, 5$, and $145 \mathrm{~kJ} \mathrm{~mol}^{-1} \mathrm{~K}^{-1}$, respectively, and $R$ is the universal gas constant $\left(8.314 \mathrm{~J} \mathrm{~mol}^{-1} \mathrm{~K}\right)$. The constants were determined by fitting equation [1] to flow stress data in the DEFORM v10.0 database. ${ }^{[15]}$

The shear friction between the deforming material and extrusion tooling (i.e., ram, container, die, and feeder plate) was defined with a constant interfacial shear stress approach,

$$
f=m \tau
$$

where $f$ is the shear force per unit area (MPa) at the interface, $\tau$ is the shear stress (MPa) of the material in contact with the tooling, and $\mathrm{m}$ is the shear factor. A shear factor of $m=1$, (i.e., complete sticking) was defined for the interface between the billet and the tooling following the work done by Schikorra et al. ${ }^{[16]}$ Inside the bearing where a shear factor of $m=0.8$ was used based on the previous study by Jowett et al. ${ }^{[17]} \mathrm{A}$ heat transfer coefficient of $25 \mathrm{~kW} \mathrm{~m}{ }^{-2} \mathrm{~K}^{-1}$ was used at the billet/tooling interfaces as suggested by Duan and Sheppard. ${ }^{[9]}$

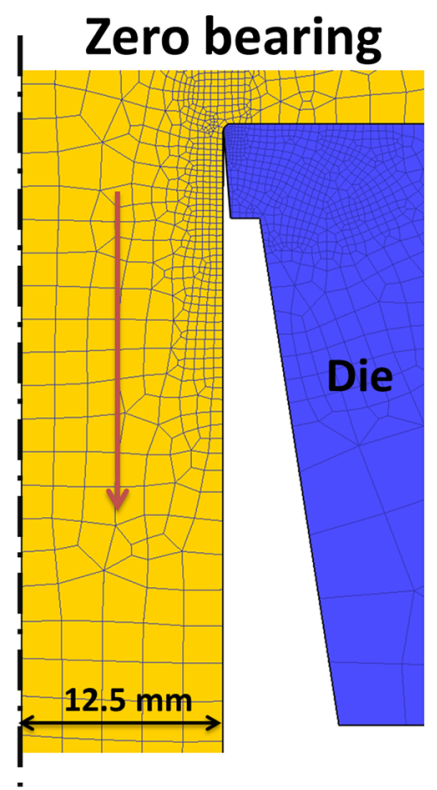

(a)

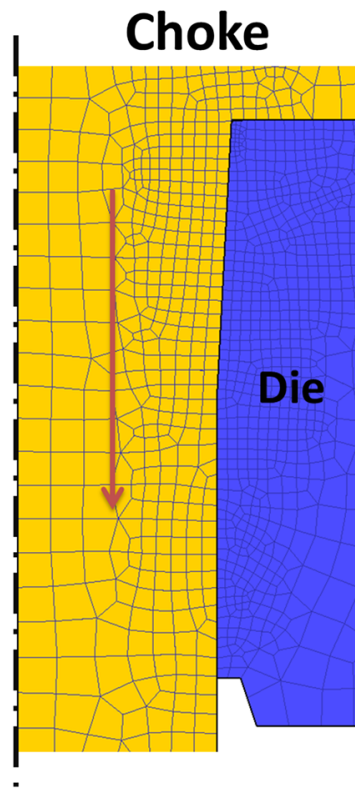

(b)
Fig. 3-DEFORM 2D FE model geometry and mesh resolution for the two die bearing geometries: $(a)$ zero bearing and $(b)$ choke die.

The stored energy in the material during extrusion was assumed primarily to arise from dislocation accumulation and the associated elastic strain energy. The flow stress, $\sigma$, is related to the dislocation density by the Taylor equation, ${ }^{[18]}$ i.e.,

$$
\sigma=\alpha_{1} M G b \sqrt{\rho}
$$

where $\alpha$ is a constant $(\approx 0.3), M$ is the Taylor factor (3.06 for random crystallographic texture) and where $G$ is the shear modulus and $b$ is the magnitude of the Burgers vector of aluminum at the deformation temperature. At the temperatures and strain rates of interest, the rate of accumulation of dislocations and the rate of dislocation annihilation are equal (dynamic recovery) in AA6082 such that a steady-state flow stress is achieved. ${ }^{[19]}$ The elastic energy per line length of dislocation is given by

$$
E_{1}=\alpha_{2} G b^{2}
$$


where $\alpha_{2}$ is a constant $(\approx 0.5)$. The dislocation density can be estimated from the flow stress by rearranging Eq. [3] and the volumetric stored energy can be determined by the product of the dislocation density and the elastic energy per line length, ${ }^{[20]}$ i.e.,

$$
E=\alpha_{2} G b^{2}\left(\frac{\sigma}{\alpha_{1} M G b}\right)^{2}
$$

or after grouping constants and noting that $\alpha_{1} M$ is $\approx 1$, one obtains ${ }^{[11]}$

$$
E=\alpha_{2} \frac{\sigma^{2}}{G}
$$

where $\alpha_{2}$ is a material constant of the order of 0.5 and $\sigma$ is the steady-state flow stress (MPa) of the material at a given temperature and strain rate calculated using Eq. [1]. The final stored energy of the material was assumed to be the maximum value of stored energy calculated for each position as the material passes through the die.

\section{RESULTS AND DISCUSSION}

\section{A. Experimental Results}

Figure 4 compares the anodized optical images for transverse sections of rods extruded at $480{ }^{\circ} \mathrm{C}$ with a ram speed of $20 \mathrm{~mm} / \mathrm{s}$ at a mid-length position. A recrystallized surface layer (Peripheral Coarse Grain, PCG) of approximately $300 \mu \mathrm{m}$ was observed for the extrusion using the zero bearing die while there was no evidence of a recrystallized surface layer for the choked die, consistent with the earlier results of Parson et al. ${ }^{[10]}$

Figure 5 shows EBSD images of longitudinal sections from the front and back locations extruded at $480{ }^{\circ} \mathrm{C}$ with a ram velocity of $20 \mathrm{~mm} / \mathrm{s}$ for the two die geometries. Figures 5(a) and (c) show the surface microstructures for the zero bearing die at the back
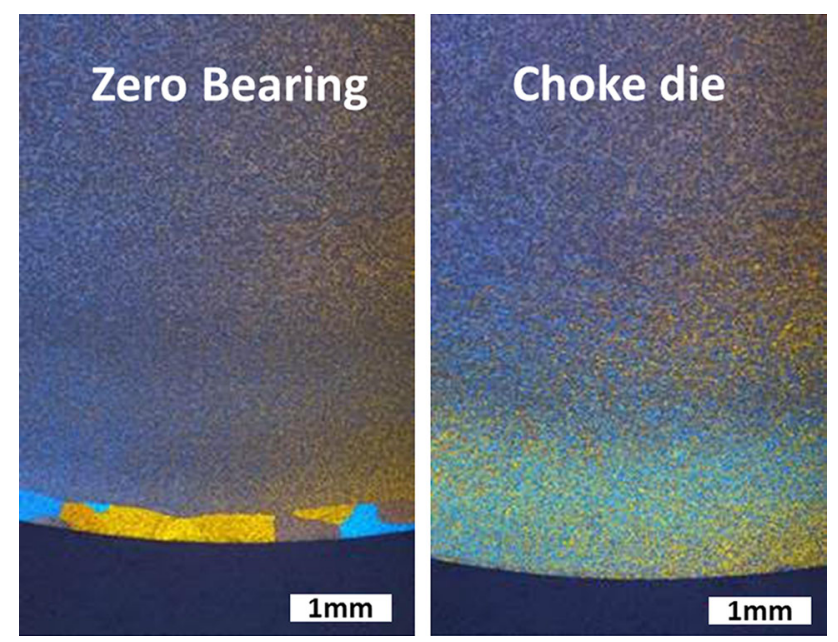

Fig. 4-Transverse grain structures from zero bearing (left) and choke bearing (right). Mid-length location, extrusion temperature $480{ }^{\circ} \mathrm{C}$, and ram speed $20 \mathrm{~mm} / \mathrm{s}$. and the front of the extrusion, respectively. A PCG layer $\sim 300 \mu \mathrm{m}$ thick containing grains oriented in the extrusion direction (red and blue) is evidently suggesting that the recrystallized grains grew along the extrusion direction. Below the PCG layer, the structure shows highly elongated grains consistent with an unrecrystallized structure. In contrast, the rods extruded through the choke die bearing show no evidence of a PCG layer at either the front or back of the extrudate (Figures 5(b) and (d), respectively) consistent with the observations from optical metallography in Figure 4. The surface of these bars exhibits a crystallographic texture consistent with a simple shear texture ${ }^{[19,20]}$ as measured by Chen. ${ }^{[21]}$

Referring to Figure 2, it can be seen that the front and back samples have different temperature histories as the front part of the extrudate passes through the quench bath but the back of the extrudate stops between the die exit and quench bath and cools more slowly. Although a fibrous unrecrystallized structure is evident for both the front and back samples away from the surface, the fibrous structure is more pronounced in the front samples for both the choke and zero bearing dies, consistent with different thermal histories between these locations, i.e., the back location spent more time at a high temperature such that the deformed structure had more opportunity for microstructure evolution by static recovery.

Figure 6(a) shows the macrostructure of a partially extruded billet along with a flow line predicted from the point tracking technique in the FEM model. ${ }^{[1]}$ The orange line shows the flow path of the material that ends up at a location approximately $100 \mu \mathrm{m}$ below the surface in the final extruded rod. Figure 6(b) shows a series of Inverse Pole Figure (IPF) EBSD microstructure maps for the zero and choked bearing extrusions, at five

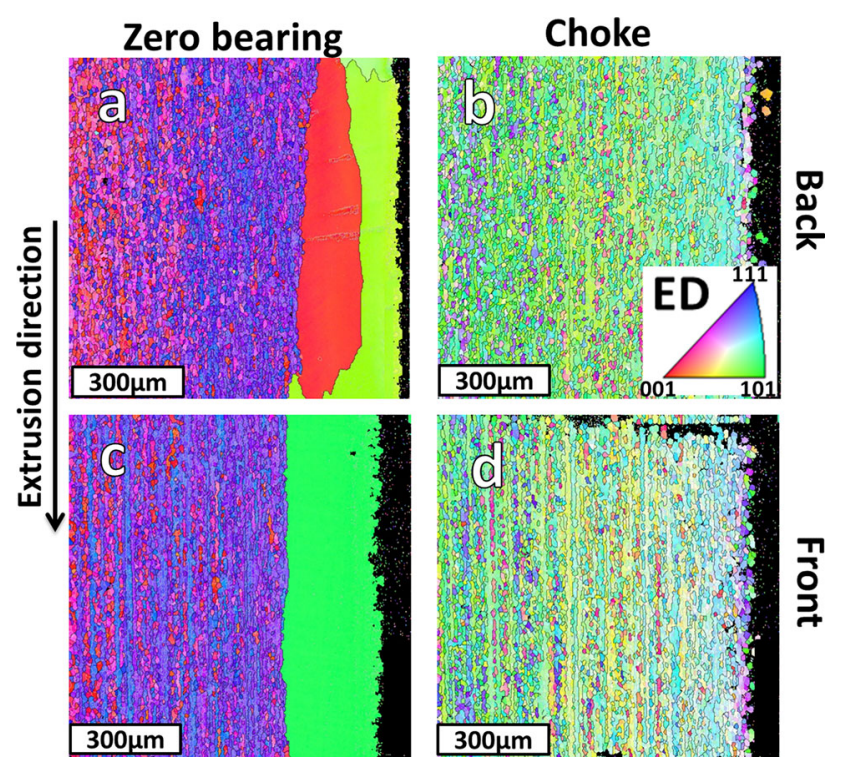

Fig. 5-Inverse pole figure (IPF) EBSD images for the longitudinal surface sections; (a) zero bearing - back, (b) choke bearing - back, (c) zero bearing - front, and (d) choke bearing - front. Extrusion temperature $480{ }^{\circ} \mathrm{C}$, ram speed $20 \mathrm{~mm} / \mathrm{s}$. 


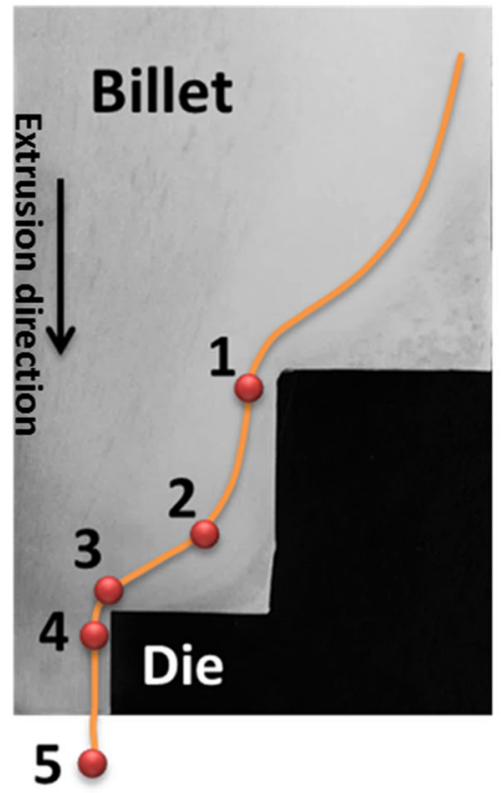

(a)

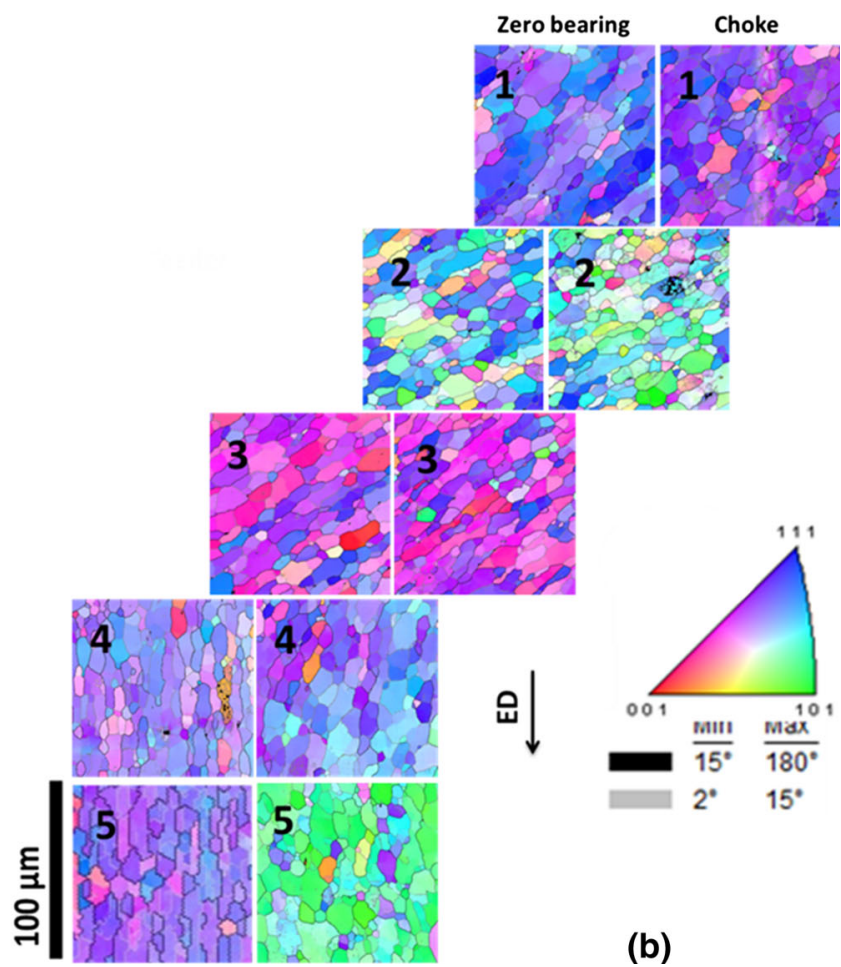

Fig. 6-(a) Location of streamline which exits the die $100 \mu \mathrm{m}$ below the surface and (b) IPF EBSD maps at positions 1 to 5 for the zero bearing die (left) and choked bearing die (right). The extrusion temperature was $480{ }^{\circ} \mathrm{C}$ and the ram speed $20 \mathrm{~mm} / \mathrm{s}$.

locations along the flow line, starting at the entry to the feeder plate and finishing after exiting the die bearing. There are no significant differences between the microstructures (grain size, grain shape, and orientation) produced using the two dies, prior to material entering the die bearing zone. In particular, the variation of the crystallographic texture as represented by the color based on the IPF scheme is similar from point 1 to point 3 for both dies. At point 4 which is located immediately after the die corner there is minimal difference in the texture but at point 5 , located after the bearing, a significant difference is observed for the two geometries. This difference can be attributed to the extra deformation applied at the surface of the extrusion by the choked bearing while with the zero bearing, the material has actually exited the die at point 4 and no further deformation occurs between points 4 and 5 .

Figure 7 shows the evolution of texture using inverse pole figure EBSD maps of partially extruded billets near the die corner area for the zero and choked bearing dies. For the material extruded using the zero bearing die, there is no evidence of deformation at the surface after the material passes the die corner (Figure 7(a)) while the color changes at the surface along the choked bearing (Figure 7(b)) indicate that the texture orientation is changing as a result of deformation imposed from friction with the choked bearing surface.

Referring to Figure 7(a), it is evident that there is no PCG for the zero bearing extrusion in the quenched partial extrusion even though a PCG layer was observed at both front and back of the extrudate for this die geometry (Figures 5(a) and (c)). An explanation for the

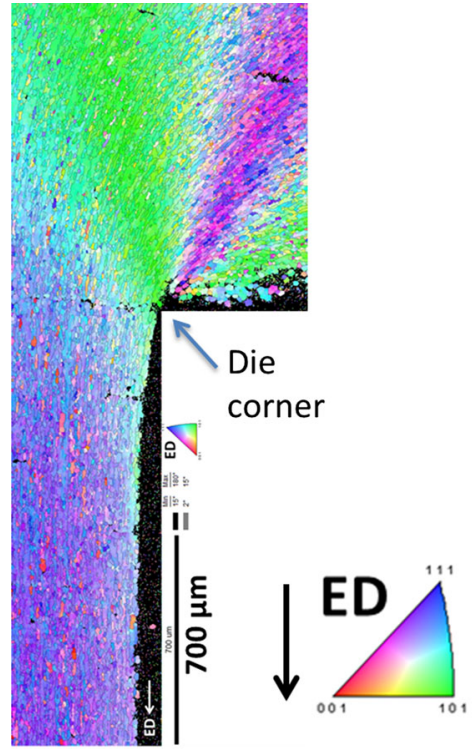

Zero bearing

(a)

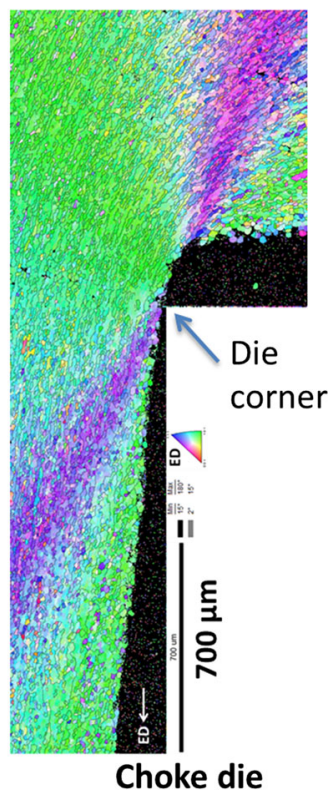

(b)
Fig. 7-IPF EBSD maps of partially extruded billets at the die corner area for $(a)$ zero bearing and $(b)$ choked die. Extrusion temperature $480{ }^{\circ} \mathrm{C}$, ram speed $20 \mathrm{~mm} / \mathrm{s}$.

absence of a PCG layer in the partially extruded billet may be related to the lower stored energy at this location due to the deceleration of the ram at the end of extrusion. In order to test this hypothesis, an extrusion trial was conducted where the ram was "dead" stopped 


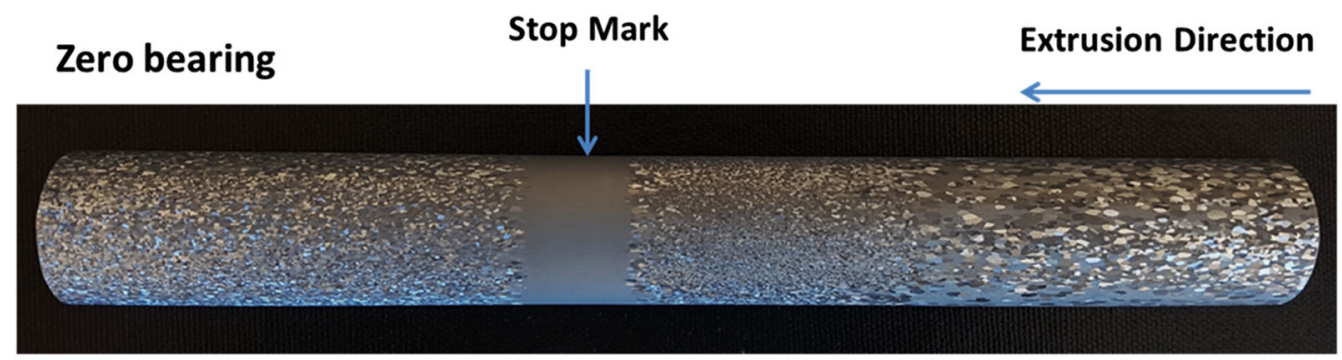

\section{Stop Mark}

(a)

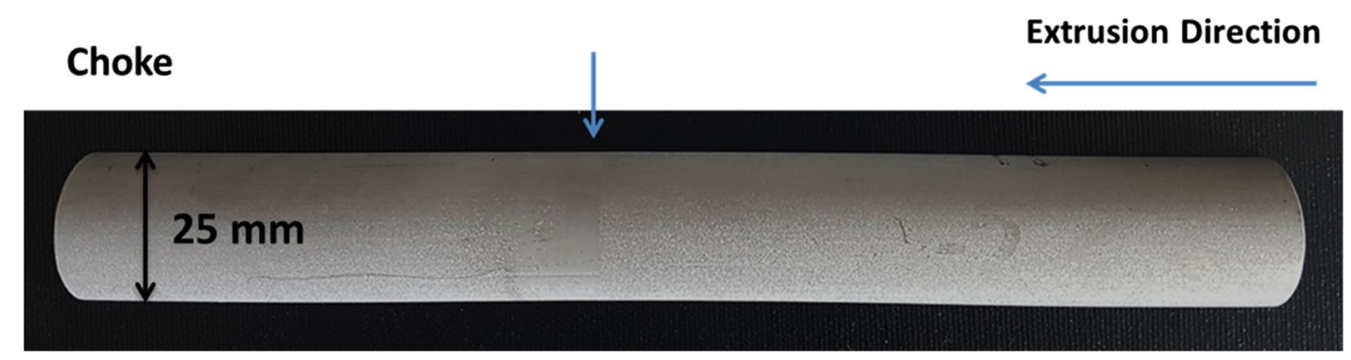

(b)

Fig. 8-Extruded surface grain structures associated with stop marks, $(a)$ zero bearing and $(b)$ choke die. (Poulton's reagent).

part way through the extrusion for 5 seconds and then restarted. Figure 8 shows the surface microstructure from this trial for both dies after macroetching using Poulton's reagent. In both cases, there is a noticeable stop mark region associated with the natural deceleration and acceleration inherent in the hydraulic system. For the case of the zero bearing die, the large surface grains (i.e., PCG) are present before and after the press stop but disappear around the stop mark as shown in Figure 8(a) (note, in this region the grain size is very small and one cannot see individual grains at this magnification, hence the appearance as a homogeneous dark gray region). This would be consistent with the suggestion that the deceleration of the ram prior to quenching the billet may account for the lack of a PCG layer in the partially extruded and quenched sample shown in Figure 7(a). A detailed explanation for this phenomenon will be given in the next section using the results from the FEM model. In contrast, Figure 8(b) shows no evidence of large surface grains for the case of the choke die before, during or after the stop. These observations are consistent with the lack of a PCG layer in Figure 7.

\section{B. Finite Element Method Model Predictions}

Figure 9 plots the experimentally measured and predicted extrusion loads at break-through for the two die bearing geometries. The difference between the predicted and measured loads is less than 5 pct; however, it is found that the $35 \mathrm{~mm}$ choke die required $\approx 15$ pct higher extrusion force compared to the zero bearing die and this trend is captured by the finite element model predictions.

Figure 10 shows model predictions of the effect of bearing geometry on extrusion exit temperature for

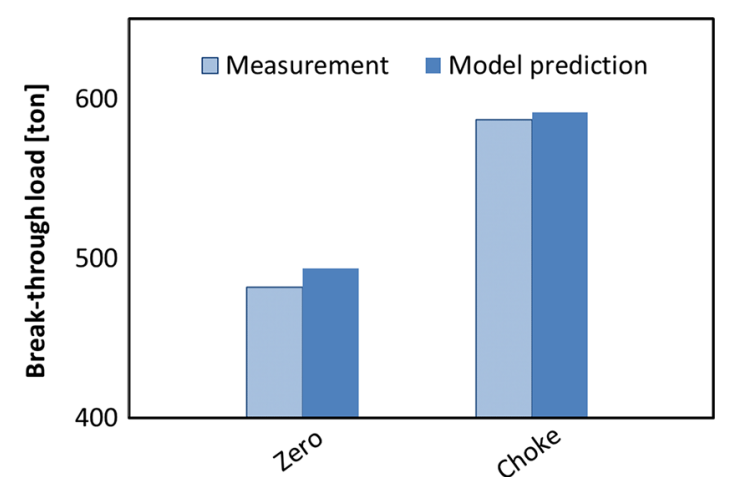

Fig. 9-Measured and model predicted break-through load as a function of the die bearing geometry. Extrusion billet, container, and die initial had an initial temperature of $480{ }^{\circ} \mathrm{C}$ and the ram velocity was $30 \mathrm{~mm} / \mathrm{s}$.

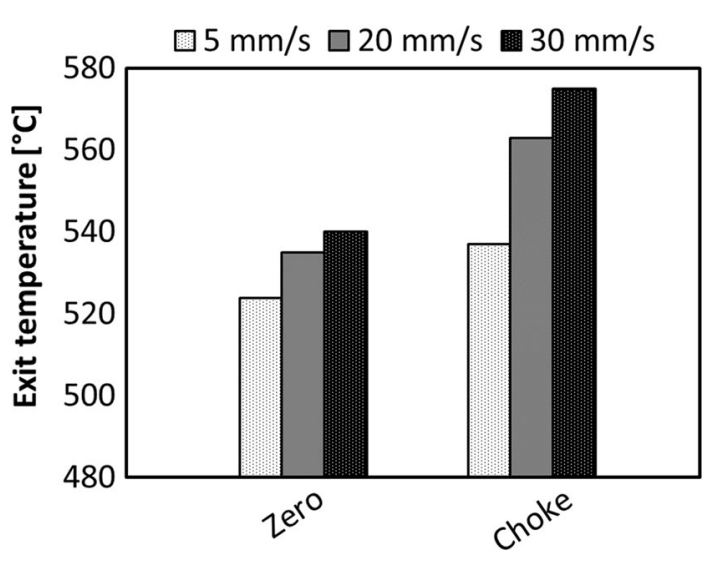

Fig. 10-Predicted exit temperature for different bearing geometries and ram velocities. The extrusion billet, container, and die initial had an initial temperature of $480{ }^{\circ} \mathrm{C}$. 
three ram velocities. The exit temperature here represents the average temperature over the cross-section of the extruded bar after the core and surface temperature have equilibrated. It is clear that the die geometry has a significant effect on the temperature rise with the choke die producing significantly higher exit temperatures. This difference in exit temperature for the two bearing geometries is accentuated as the ram velocity is increased.

Figure 11 shows the effect of die exit geometry on the distribution of temperature and strain rate near the die bearing region. The choke die results in higher surface temperatures due to the increased heating from friction and surface strain imparted in the bearing region. The longer the bearing length and exposure to this friction, the higher the surface temperature becomes and the deeper the temperature can penetrate into the subsurface region. It is noted that the FEM predictions for the temperature at the surface of choke die extrusion are above the solidus point of the alloy (estimated to be $590{ }^{\circ} \mathrm{C}$ using Thermocalc with the TTAl6 database). However, local melting requires an additional energy input (the latent heat of fusion) which resists melting. This was not accounted for in the finite element method. In addition, the material spends a very short time at the maximum temperature due to the high deformation speed. Temperature measurements taken after die exit were always below the melting point (see Figure 10) and there was no microstructural evidence of melting or speed cracking on the surface. Thus, we do not think that the local melting occurred in these experiments.

The bearing geometry also has an effect on the strain rate distribution. For the zero bearing, there is no further deformation once the material passes the corner of the die since there is no more contact between the die and the extrudate. In contrast, with the choke die, the surface strain rate is higher than at the core and

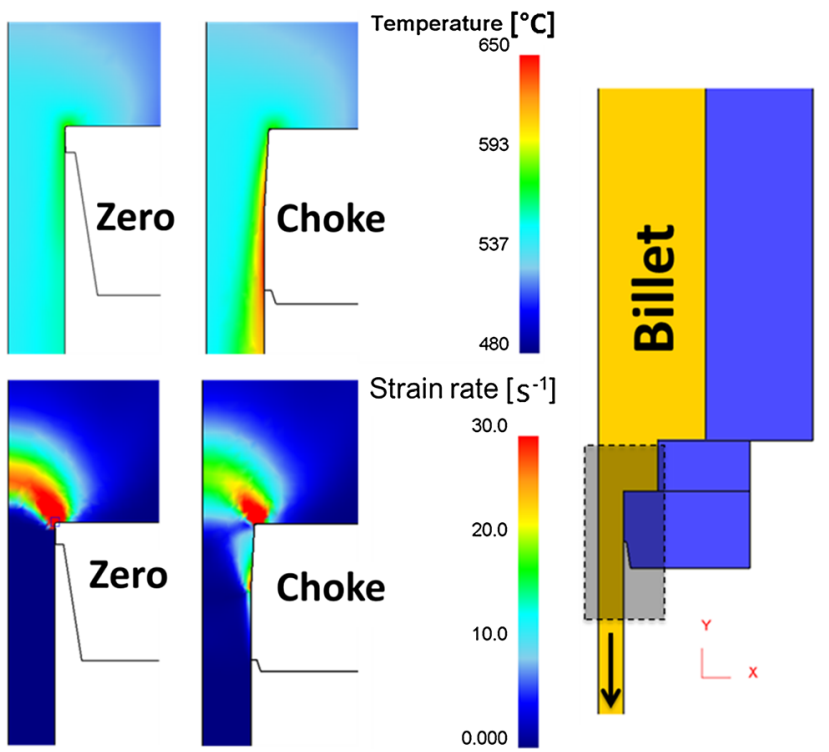

Fig. 11-Predicted temperature and strain rate distributions for both die bearing geometries. Billet, container, and die initial temperature of $480{ }^{\circ} \mathrm{C}$, ram velocity $20 \mathrm{~mm} / \mathrm{s}$. penetrates a significant distance into the extrudate due to the decreasing radius of the die in the choke zone. In the following section, it will be demonstrated that the details of these variations in temperature and strain rate can result in different levels of stored energy in the extrudate and can influence the subsequent microstructure evolution.

Figure 12 shows the spatial distribution of maximum stored energy for the two die geometries calculated in the FEM code using Eq. [3]. This estimate for stored energy is $\sim 5$ pct lower for the choke die compared to the zero bearing at all locations across the extrudate radius which is a possible explanation for the presence of a PCG layer with the zero bearing and the absence of such a layer for the choke die.

As described above, the effect of strain rate was examined by performing a "dead" stop of the ram at the midpoint of the extrusion. Figure 13 shows the actual ram velocity/time profile measured on the extrusion press during the deceleration, stop and restart of the ram, compared with the profile used in the finite element simulation.

Figure 14 compares the finite element simulation estimates for the stored energy (top image) and the

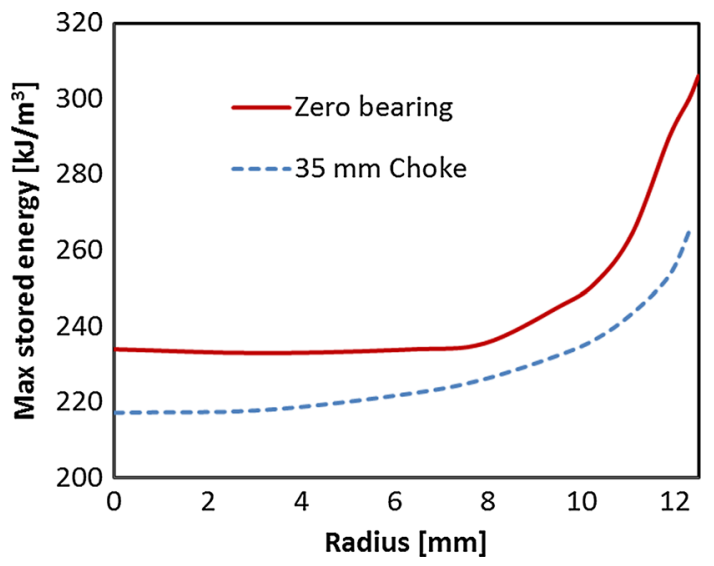

Fig. 12 - Calculated stored energy distribution across the extrudate radius for both die bearing geometries. Extrusion temperature $480{ }^{\circ} \mathrm{C}$, ram speed $20 \mathrm{~mm} / \mathrm{s}$.

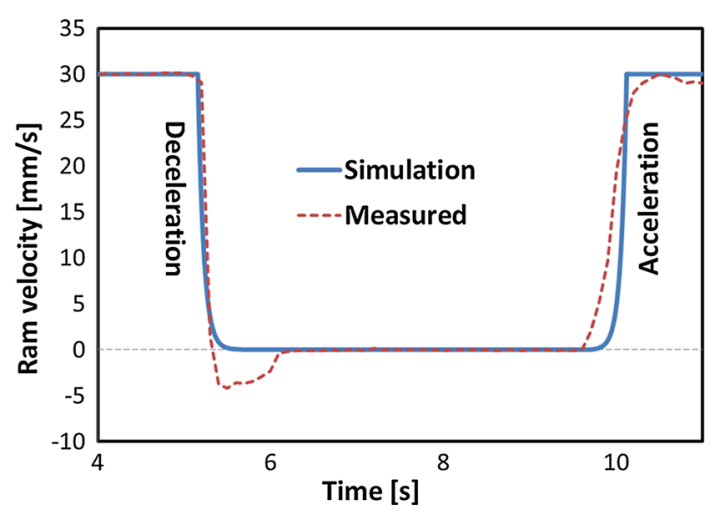

Fig. 13-Experimentally measured and simulated ram velocity as a function of time near the stop point. 
maximum surface temperature (bottom image) with the experimentally observed extruded surface macrostructure at the region around the stop point for the zero bearing die (noting again that the dark, homogeneous region around the stop mark corresponds to fine surface grains which cannot be resolved at this magnification). The banding effect in the color of the calculation of the stored energy on the right and left sides of the stop line is believed to be a numerical artifact from the simulations. Having noted this, it can be seen that the region in the macrograph around the stop mark where the large surface grains are absent corresponds to the area where the estimate for stored energy is the lowest (green in the top image). On the other hand, the location of the lowest surface temperature (light green in the bottom image) is not aligned with the fine grain region at the stop mark and actually corresponds to an area where large surface grains are observed experimentally.

To further examine the distribution of stored energy around the stop point, Figure 15 plots a longitudinal section through the center of the extrudate for the zero bearing die. Consistent with Figure 14, these results show that the surface region at the stop mark where no large surface grains are observed corresponds closely with the region where the predicted surface stored energy is at a minimum. To examine the $3 \mathrm{D}$ spatial distribution of stored energy, temperature, and equivalent plastic strain, Figure 16 shows snapshots from the simulations around the stop mark for the zero bearing die where a quarter of the geometry has been removed.

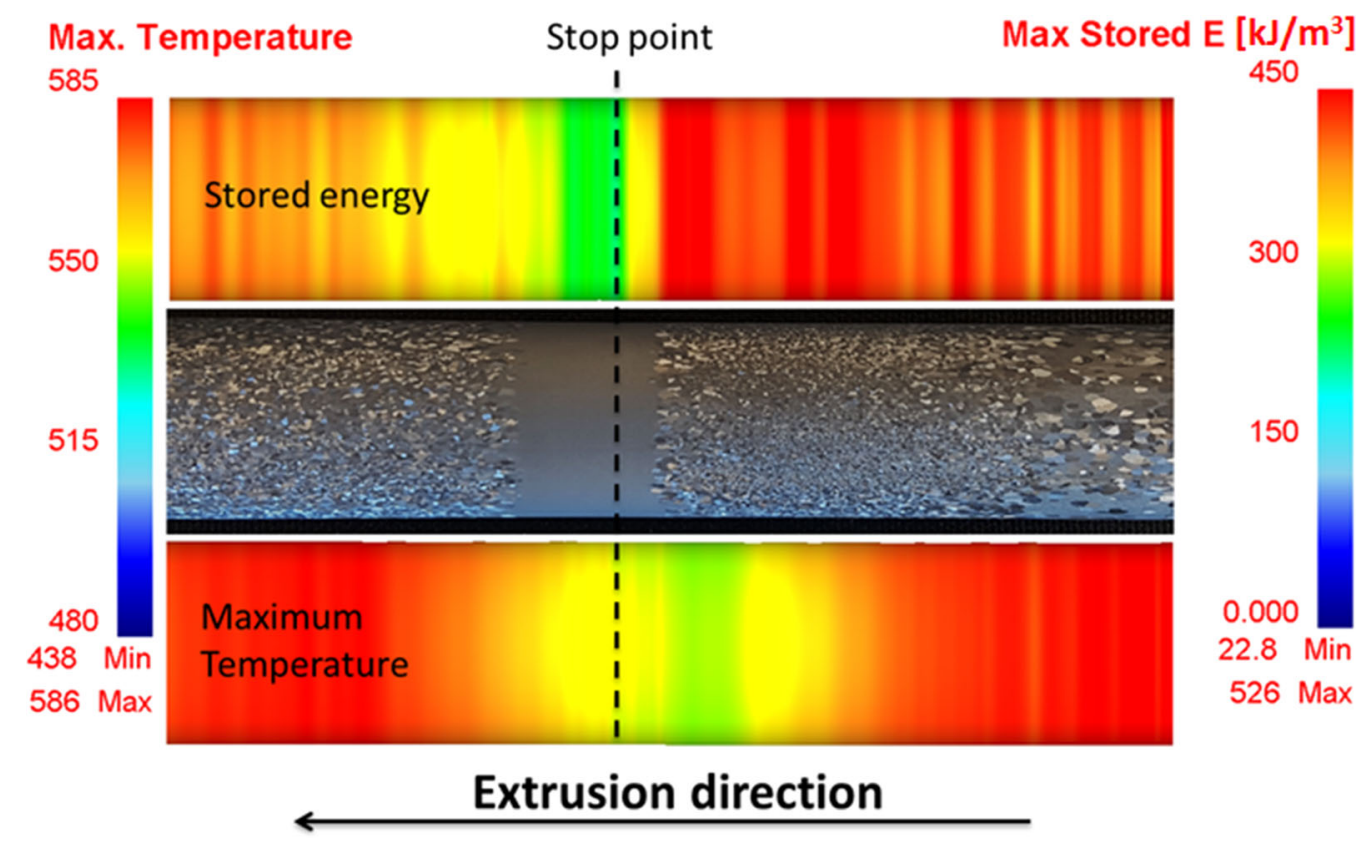

Fig. 14 Comparison of predicted surface stored energy (top) and maximum surface temperature (bottom) distributions with surface grain structure. Zero bearing die.

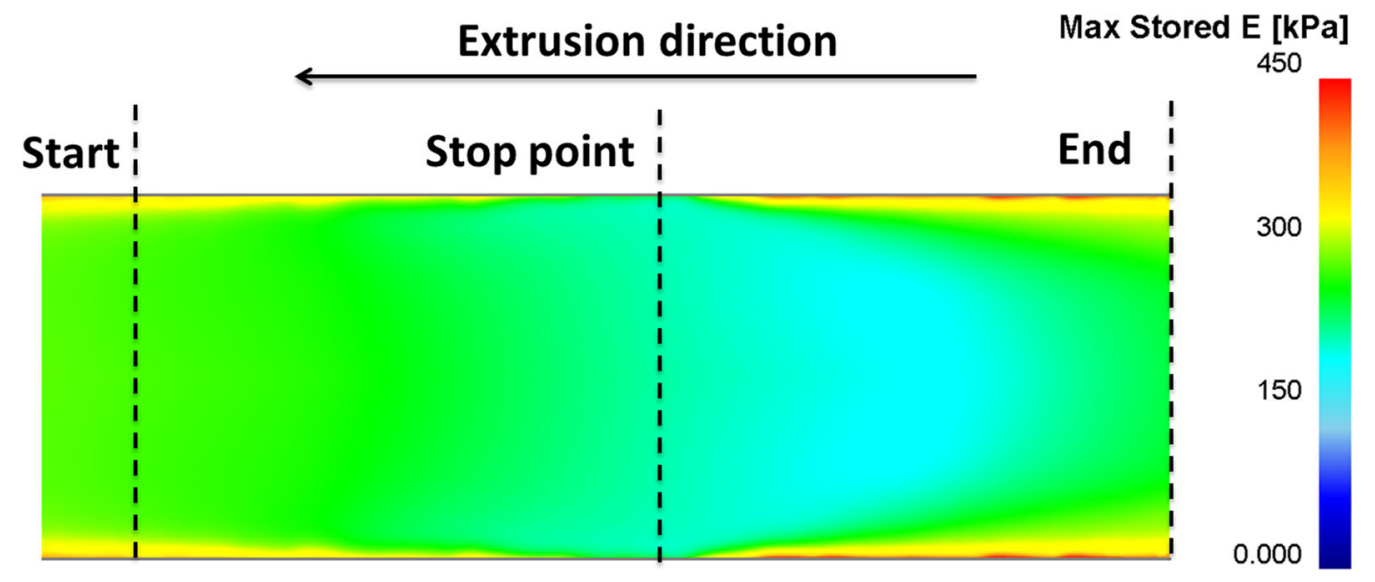

Fig. 15-Predicted stored energy distribution through the center of the extrudate around the stop point, zero bearing die. 


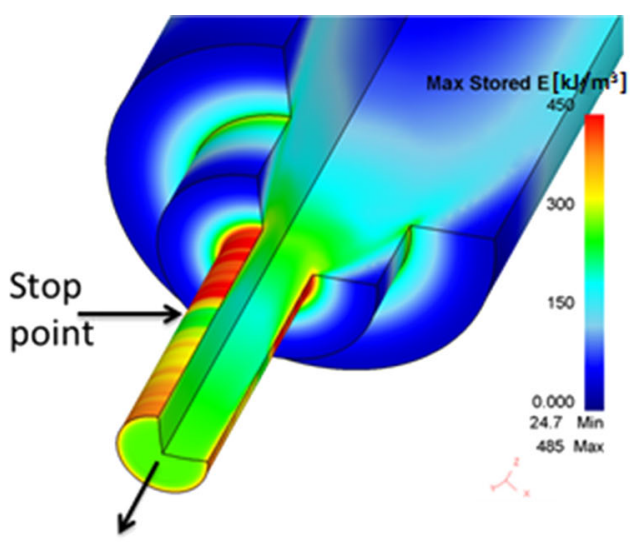

(a)

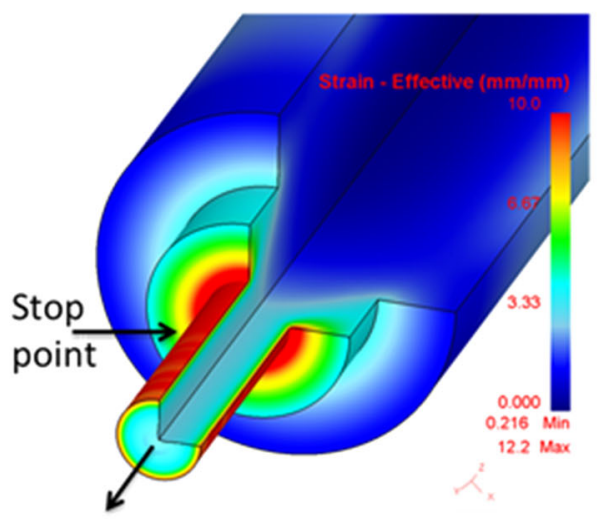

(c)

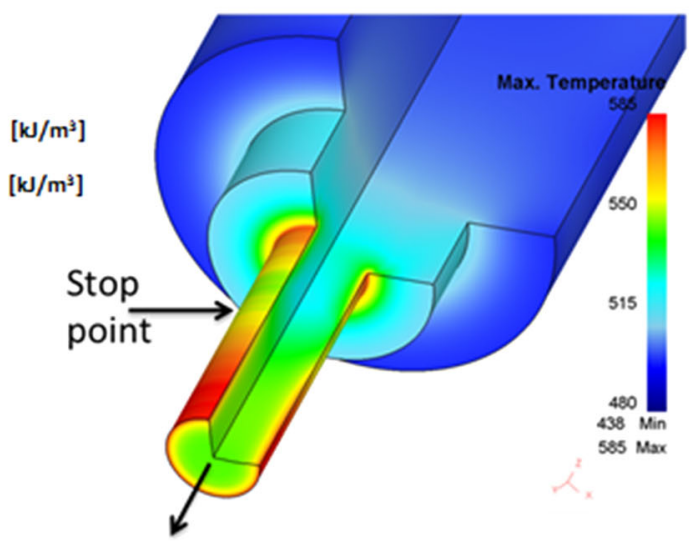

(b)

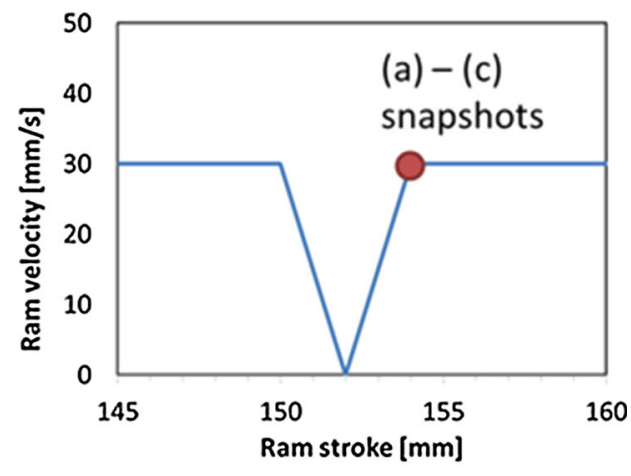

(d)

Fig. 16-Predicted distributions of $(a)$ local stored energy, $(b)$ maximum temperature, and $(c)$ accumulated plastic strain, around the stop point for interrupted extrusion trial with zero bearing die with a velocity change profile shown in (d). The snapshots shown in (a), (b), and (c) correspond to the location shown in (d) corresponding to the point (red circle) where the original velocity was restored.

Here, it can be seen that the estimated stored energy (Figure 16(a)) and temperature (Figure 16(b)) distributions change as the extrusion velocity decreases, stops, and then accelerates back to the original velocity. In contrast, the distribution of plastic strain does not depend on the extrusion velocity as this is essentially kinematically constrained by the geometry of the die. Given the previously described spatial correlation between local stored energy and the absence of large surface grains, this suggests that local stored energy is one of the key factors controlling recrystallization and grain growth at the surface of extruded materials. However, it is worth noting here that even though the stored energy calculation using Eq. [3] is quite simplistic (i.e., it does not include details on the complexity of the deformed state dislocation density, subgrain structure, high-angle boundary structure, dispersoid size, and volume fraction), it does correlate well with the experimental observations.

Finally, returning to previous studies, the current results are consistent with the mechanism proposed by Van Geertruyden et al. ${ }^{[22]}$ where the surface grains undergo very large shear strains due to friction between the billet and die leading to continuous dynamic recrystallization (CDRX) or geometric dynamic recrystallization (GDRX) to produce a surface region with very fine grains (of order 1 to $2 \mu \mathrm{m}$ in diameter). The GDRX mechanism is more likely given that the surface strain is $>10$ for the current case as shown in Figure 16(c). These microstructures and their local stored energy are sensitive to the conditions under which they formed. Given a sufficiently high stored energy and temperature, abnormal grain growth can occur resulting in a PCG layer. It is clear from the current experiments that there is a fine balance between the local stored energy and the temperature which determines whether abnormal grain growth occurs or not. For example, reducing the extrusion velocity or adding a choke to the die exit, both suppress the formation of the large surface grains. This suggests that careful design of the die and the extrusion process can minimize the formation of undesirable, coarse, surface grains.

\section{SUMMARY}

The current study has considered the effect of bearing die geometry on the microstructure development at the surface of an Al-Mg-Si-Mn extrusion alloy for two 
limiting die exit geometries (zero bearing and choke). It was shown that the die bearing geometry has a strong impact on the thermomechanical history of the material close to the surface of the extrudate and therefore the resulting surface microstructure. It was found that a layer of large surface grains (PCG) was produced using a zero bearing die, whereas the choke die prevented PCG layer formation. In detail, the main results of the current work are as follows:

1. EBSD studies on partially extruded and quenched samples provided insight into how the microstructure developed passing through both die configurations. The subsurface microstructure was found to evolve in a very similar manner for the two geometries up until the aperture or die bearing entry point, indicating that the difference in surface microstructure resulted from variations in the details of how the material exits the die, i.e., with or without the choke.

2. Experimental observations on material that was water quenched in die and then examined by EBSD found that a PCG layer was not observed for either die geometry in contrast to the steady-state situation. It was hypothesized that this was related to the deceleration of the extrusion ram at the press stop prior to quenching. This hypothesis was experimentally investigated by "dead" stopping the extrusion ram part way through the extrusion process, holding for 5 seconds and then restarting the extrusion. Indeed, this experiment showed that the PCG layer disappeared in the zero bearing die as the extrusion ram speed decreased and stopped. Upon restarting the extrusion process, the PCG layer reappeared once the steady-state ram speed was achieved.

3. Finite element method calculations were conducted to simulate the steady-state and "dead" stop extrusion experiments. For the steady-state extrusion experiment, the FEM predictions for the breakthrough pressure were within 5 pct of the measured values. For the "dead" stop extrusion trials, the FEM calculations indicated that both the local stored energy at the surface and the surface temperature decreased as the extrusion was slowed and then stopped. However, the location of the refined microstructure zone relative to the die stop line closely correlated with the stored energy calculations but was not aligned with the region of lowest temperature. As such, it is proposed that it is the local stored energy at the surface of the extrudate which controls the stability of the deformed structure.

In conclusion, the current study demonstrated that changes in the extrusion velocity or the design of the die bearing influence large surface grains formation, suggesting that this behavior is very sensitive to the details of the deformed state at the surface of the extrudate. It is proposed that a combination of finite element simulations and a simple estimate of the local stored energy can be used to predict the formation of the PCG layer. However, it is noted that the sensitivity of this phenomenon to relatively small changes in process conditions may make this challenging to implement in industrial settings. Finally, although the conclusions from this paper are best suited for simple geometry solid profiles such as bars, rods and strips, including forging stock, the principles can be applied to more complex, thin, and hollow profiles.

\section{ACKNOWLEDGMENT}

The authors would like to gratefully acknowledge the financial support of Rio Tinto Aluminium and NSERC Canada (Natural Sciences and Engineering Research Council of Canada).

\section{OPEN ACCESS}

This article is distributed under the terms of the Creative Commons Attribution 4.0 International License (http://creativecommons.org/licenses/by/4.0/), which permits unrestricted use, distribution, and reproduction in any medium, provided you give appropriate credit to the original author(s) and the source, provide a link to the Creative Commons license, and indicate if changes were made.

\section{REFERENCES}

1. N. Parson, S. Barker, A. Shalanski and C. Jowett: Proceedings of the 8th International Aluminum Extrusion Technology Seminar, 2004, Orlando, FL.

2. L. De Peri Jr and W.J. Misiolek: Acta Mater., 2008, vol. 56, pp. 6174-85.

3. A.R. Eivani: PhD thesis, Delft University of Technology, 2010.

4. W.H. Van Geertruyden, H.M. Brown, W.Z. Misiolek, and P.T. Wang: Metall. Mater. Trans. A, 2005, vol. 36A, pp. 1049-56.

5. P. Saha: Aluminum Extrusion Technology, ASM International, Ohio, 2000.

6. C.L. Liu, Q. Du, N.C. Parson, and W.J. Poole: Scripta Mater., 2018, vol. 152 , pp. 59-63.

7. I. Charit and I.S. Mishra: Scripta Mater., 2008, vol. 58, pp. 367-71.

8. M. Jarrett and N.C. Parson: UK Patent GB2285941, 1995.

9. X. Duan and T. Sheppard: Mater. Sci. Eng. A, 2003, vol. 351, pp. 282-92.

10. N. Parson, A. Maltais and C. Jowett: Proceedings of Tenth International Aluminum Extrusion Technology Seminar (ET'12), 2012, Miami, FL.

11. Y. Mahmoodkhani: $\mathrm{PhD}$ thesis, University of Waterloo, Waterloo ON, Canada, 2013.

12. C. Liu: PhD thesis, University of British Columbia, Vancouver, BC, Canada, 2017.

13. Y. Mahmoodkhani, M.A. Wells, N. Parson, and W. Poole: $J$. Mater. Process. Technol., 2014, vol. 214 (3), pp. 688-700.

14. H.J. McQueen and N.D. Ryan: Mater. Sci. Eng. A, 2002, vol. 322, pp. 43-63.

15. DEFORM v10.0 Database.

16. M. Schikorra, L. Donati, L. Tomesani, and A.E. Tekkaya: J. Mech. Sci. Technol., 2007, vol. 21, pp. 1445-1451.

17. C. Jowett, Y. Mahmoodkhani, N. Parson and G. Garza: Eleventh International Aluminum Extrusion Technology Seminar (ET '16), 2016, Chicago, IL.

18. F.J. Humphreys and M. Hatherly: Recrystallization and Related Annealing Phenomena, 2nd ed., Elsevier, Amsterdam, 2004. 
19. F. Montheillet, M. Cohen, and J. Jonas: Acta Metall., 1984, vol. 32, p. 2077

20. G.R. Canova, U.F. Kocks, and J.J. Jonas: Acta Metall., 1984, vol. 32 , p. 211

21. J. Chen: PhD thesis, The University of British Columbia, Vancouver BC, Canada, 2018.
22. W.H. van Geertruyden, W.Z. Misiolek, and P.T. Wang: J. Mater. Sci., 2005, vol. 40, pp. 3861-63.

Publisher's Note Springer Nature remains neutral with regard to jurisdictional claims in published maps and institutional affiliations. 